\title{
Structure, stability, and reorganization of 0.5 LO topography in block copolymer thin films
}

Article

Accepted Version

Maher, M. J., Self, J. L., Stasiak, P., Blachut, G., Ellison, C. J., Matsen, M. W., Bates, C. M. and Willson, C. G. (2016)

Structure, stability, and reorganization of 0.5 LO topography in block copolymer thin films. ACS Nano, 10 (11). pp. 1015210160. ISSN 1936-086X doi:

https://doi.org/10.1021/acsnano.6b05390 Available at https://centaur.reading.ac.uk/67474/

It is advisable to refer to the publisher's version if you intend to cite from the work. See Guidance on citing.

To link to this article DOI: http://dx.doi.org/10.1021/acsnano.6b05390

Publisher: American Chemical Society

All outputs in CentAUR are protected by Intellectual Property Rights law, including copyright law. Copyright and IPR is retained by the creators or other copyright holders. Terms and conditions for use of this material are defined in the End User Agreement. 


\section{CentAUR}

Central Archive at the University of Reading

Reading's research outputs online 


\section{Structure, Stability, and Reorganization of $0.5 L_{0}$}

\section{Topography in Block Copolymer Thin Films}

Michael J. Maher, ${ }^{1,5 \ddagger}$ Jeffrey L. Self, ${ }^{, 3 \ddagger}$ Pawel Stasiak, ${ }^{4 \ddagger}$ Gregory Blachut, ${ }^{2}$ Christopher J. Ellison, ${ }^{2,5^{*}}$ Mark W. Matsen, ${ }^{6 *}$ Christopher M. Bates, ${ }^{7,8^{*}}$ and C. Grant Willson ${ }^{1,2^{*}}$

${ }^{1}$ Department of Chemistry and the ${ }^{2}$ McKetta Department of Chemical Engineering, The University of Texas at Austin, Austin, TX 78712

${ }^{3}$ Department of Chemistry, University of California, Santa Barbara, Santa Barbara, CA 93106.

${ }^{4}$ Department of Mathematics \& Statistics, University of Reading, Reading, U.K.

${ }^{5}$ Department of Chemical Engineering and Materials Science, University of Minnesota, Minneapolis, MN 55455

${ }^{6}$ Department of Chemical Engineering, Department of Physics \& Astronomy, Waterloo Institute for Nanotechnology, University of Waterloo, Waterloo, Ontario, Canada

${ }^{7}$ Materials Department and ${ }^{8}$ Department of Chemical Engineering, University of California, Santa Barbara, Santa Barbara, CA 93106.

\footnotetext{
* To whom correspondence should be addressed: willson@che.utexas.edu, bates@engr.ucsb.edu, mwmatsen@uwaterloo.ca, cellison@umn.edu,

‡ Denotes equal contributions
} 


\begin{abstract}
The structure, stability, and reorganization of lamella-forming block copolymer thin film surface topography ("islands" and "holes") were studied under boundary conditions driving the formation of $0.5 L_{0}$ thick structures at short thermal annealing times. Selfconsistent field theory predicts the presence of one perfectly neutral surface renders $0.5 L_{0}$ topography thermodynamically stable relative to $1 L_{0}$ thick features, in agreement with previous experimental observations. The calculated through-film structures match crosssection scanning electron micrographs, collectively demonstrating the pinning of edge dislocations at the neutral surface. Remarkably, near-neutral surface compositions exhibit $0.5 L_{0}$ topography metastability upon extended thermal treatment, slowly transitioning to 1 $L_{0}$ islands or holes as evidenced by optical and atomic force microscopy. Surface restructuring is rationalized by invoking commensurability effects imposed by slightly preferential surfaces. The results described herein clarify the impact of interfacial interactions on block copolymer self-assembly and solidify an understanding of $0.5 L_{0}$ topography, which is frequently used to determine neutral surface compositions of considerable importance to contemporary technological applications.
\end{abstract}

Keywords: Block Copolymers, Self-assembly, Islands, Holes, Terracing, Surface Reconstruction, SCFT, Thin Films

Surface topography formed in block copolymer (BCP) thin films sensitively depends upon the nature of the interactions at the substrate and top surfaces. ${ }^{1-4}$ When BCP lamellae orient parallel to the substrate, classic "island" and "hole" structures that adopt $1 L_{0}$ height 
or depth form from initially incommensurate film thicknesses,, 5 the definition of which depends on surface boundary conditions. When both surfaces display affinity for the same block ("symmetric wetting"), BCP films preferentially adopt commensurate $t=n L_{0}$ film thicknesses and avoid incommensurate thicknesses $(n+0.5) L_{0}$; islands form when $n<t_{0} / L_{0}$ $<(n+0.5)$ and holes form when $(n+0.5)<t_{0} / L_{0}<(n+1)$. (Note the distinction between $t_{0}$, the as-cast film thickness, and $t$, the film thickness adopted after annealing; $n$ is an integer and $L_{0}$ is the bulk equilibrium domain spacing.) In contrast, the use of two surfaces that favor interaction with different blocks ("asymmetric wetting") produces commensurability at $t=$ $(n+0.5) L_{0}$ and incommensurability at $t=n L_{0}$; holes form when $n<t_{0} / L_{0}<(n+0.5)$ and islands form when $(n+0.5)<t_{0} / L_{0}<(n+1)$. Recently, sufficiently thin films (roughly $t_{0} \leq 3$ $L_{0}$ ) confined by a single ostensibly neutral surface and a highly preferential surface (in either possible permutation) have been demonstrated to generate surface topography characterized by $0.5 L_{0}$ height or depth and commensurability conditions including both $t=$ $n L_{0}$ and $t=(n+0.5) L_{0} .{ }^{7}$ These relaxed commensurability constraints create an additional switch between topography at $t_{0}=(n \pm 0.25) L_{0}$; "half-height" islands form when $n<t_{0} / L_{0}<$ $(n+0.25)$ and $(n+0.5)<t_{0} / L_{0}<(n+0.75)$, while "half-depth" holes form when $(n+0.25)<$ $t_{0} / L_{0}<(n+0.5)$ and $(n+0.75)<t_{0} / L_{0}<(n+1)$. The formation of these $0.5 L_{0}$ structures, coupled with the addition of distinct commensurability conditions not seen with $1 L_{0}$ structures, provides a particularly easy methodology for screening neutral surface compositions. $^{8}$

Here, we address both the structure and stability of $0.5 L_{0}$ topography in thin films $\left(t_{0}<3 L_{0}\right)$ through a combination of theory and experiment. This report is organized into three sections: (i) the through-film configuration of $0.5 L_{0}$ topography as evidenced by self- 
consistent field theory (SCFT) calculations and cross-section scanning electron microscopy (SEM), (ii) the thermodynamic (meta)stability of $0.5 L_{0}$ topography in the presence of one preferential surface paired with either a perfectly neutral surface or a near-neutral (slightly preferential) surface, and (iii) the restructuring mechanism that transitions the film from metastable $0.5 L_{0}$ to stable $1 L_{0}$ topography in the latter case of (ii). These topics are extensively interrogated with experiments exhaustively spanning variable space including wetting conditions and film thickness. Details concerning the poly(styrene-block-4trimethylsilylstyrene) block copolymer (PS-PTMSS, $L_{0}=22 \mathrm{~nm}$ ), surfaces, and simulations subsequently elaborated are provided in the Materials and Methods section along with the Supporting Information. The fundamental insights delivered herein significantly deepen our understanding of block copolymer thin film self-assembly.

\section{Results}

Structure

SCFT was used to simulate the structure of symmetric $\mathrm{AB}$ diblock copolymer thin films confined by one preferential surface and one neutral surface; both permutations were studied (preferential top/neutral bottom and neutral top/preferential bottom) to emulate analogous experiments utilizing either a free surface or polymeric top coat.7,8 Figure 1A,B reports results obtained for a variety of as-cast thicknesses ranging from $t_{0}=0.5-2.5 L_{0}$ where $m \equiv t / L_{0}$ is defined with the thinner $t$ value adopted after annealing. Half topography is clearly formed for each film thickness, with both blocks contacting the neutral surface and a single block wetting the preferential surface. Similar data are also produced with other values of $m$ and $f_{\mathrm{A}}$ (Figures S1-S2). The neutral surface visibly pins the edge 
dislocation in either case, verifying previous speculative illustrations. ${ }^{7,8}$ Figure $1 \mathrm{C}$ shows select corresponding cross-section SEMs that are fully consistent with this prediction; the edge dislocation is never found within the interior of the film and is only located against the neutral surface, either top or bottom. (Experimental difficulties acquiring complementary data on thinner films prevent us from reporting samples with $m<2.5$, but all indications suggest comparable disposition.) The edge dislocation observed with $0.5 L_{0}$ topography fundamentally differentiates the through-film structure compared to $1 L_{0}$ islands and holes formed by two preferential interfaces. In the latter case, the defect is embedded within the interior of the film (Figures S3-S4), ${ }^{9-12}$ adopting a location determined by a delicate balance between surface field strength, commensurability, film thickness, and block copolymer attributes like volume fraction. Relatively thick films annealed with only one neutral surface experimentally yield mixed orientations as a function of through-film position (Figure S5), with perpendicular features contacting the neutral surface spatially transforming into parallel features induced by a single block wetting the preferential surface. The exact $t_{0}$ differentiating the $0.5 L_{0}$ topography and mixed morphology regimes was not investigated, but the cross-section micrographs are in qualitative agreement with thick films previously studied. ${ }^{13,14}$ 

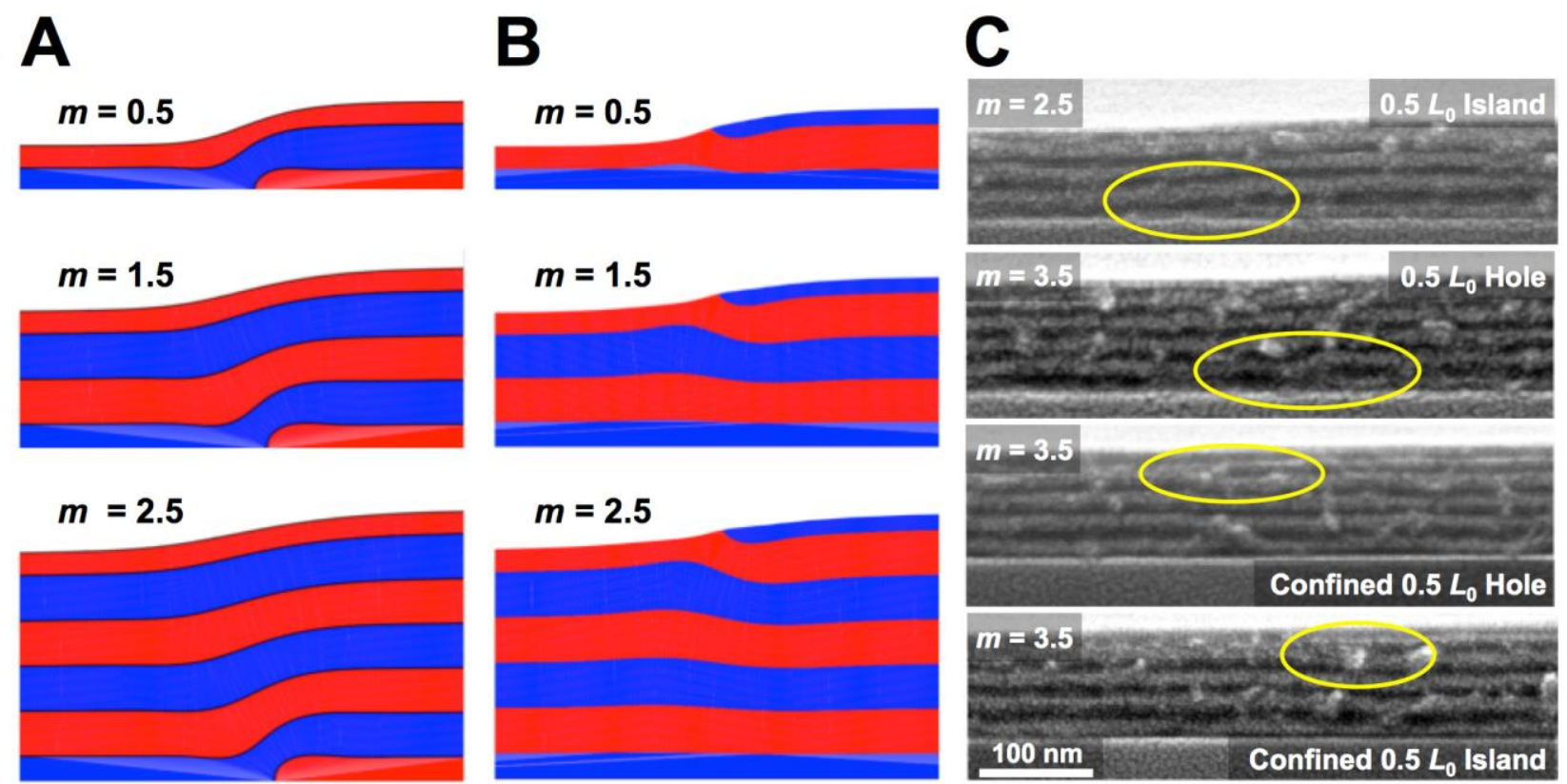

Figure 1: (A, B) Simulated step edges between films with $m$ and $m+0.5$ lamellae (i.e., $0.5 L_{0}$ topography) generated with $f_{\mathrm{A}}=0.5$ using (A) a neutral substrate surface and preferential top surface and (B) a preferential substrate surface and neutral top surface. The red and blue blocks correspond to PTMSS and PS, respectively. (C) Cross section SEMs lightly etched side-on and coated top-down with $\mathrm{Au} / \mathrm{Pd}(<3 \mathrm{~nm})$ to accentuate contrast between PS (dark) and PTMSS (light). Top coats were not removed from confined samples prior to imaging. Edge dislocation defects are highlighted by yellow circles.

\section{Stability}

The SCFT predicts that the equilibrium coexistence between terraces separated by $0.5 L_{0}$ is only possible for a perfectly neutral surface..$^{15}$ Given that the surface preference can never be precisely zero in an actual experiment, this begs the question of how the $0.5 L_{0}$ topography would ever occur. The explanation has to do with kinetics. Since the equilibrium terrace heights are separated by first-order transitions and the topography is 
stable with respect to small fluctuations, the phase separation into coexisting terraces will occur by the classical nucleation and growth mechanism. As such, the formation (i.e., nucleation) of islands or holes involves overcoming an energy barrier. A simple calculation (see Supporting Information Figure S6 and associated text) predicts that the relative barrier heights, $E_{\mathrm{B}, 0.5}$ and $E_{\mathrm{B}, 1}$, for nucleating $0.5 L_{0}$ and $1 L_{0}$ structures, respectively, on a neutral surface is given by

$$
\frac{E_{B, 0.5}}{E_{B, 1}}=2\left(\frac{\Gamma_{0.5}}{\Gamma_{1}}\right)^{2}
$$

where $\Gamma_{0.5}$ and $\Gamma_{1}$ are the line tensions of $0.5 L_{0}$ and $1 L_{0}$ step edges, respectively. The factor of 2 comes from the fact that, for a given volume of material, the shorter $0.5 L_{0}$ islands (or shallower $0.5 L_{0}$ holes) will occupy twice the area of their $1 L_{0}$ counterparts. Thus, their step edges will be longer, which impedes their nucleation. However, our SCFT predictions for the line tensions in Figure 2 shows that $\Gamma_{0.5} \approx 0.3 \Gamma_{1}$, which is more than enough to compensate for the factor 2 making the nucleation of $0.5 L_{0}$ islands (or holes) far faster than their $1 L_{0}$ counterparts. This conclusion holds for different film thickness, $m$, different diblock compositions, $f_{A}$, and regardless of whether or not it is the substrate or free surface that is neutral. Of course, the equilibrium film thicknesses are actually separated by $L_{0}$, and so the initial $0.5 L_{0}$ topography should eventually switch to $1 L_{0}$ topography. 

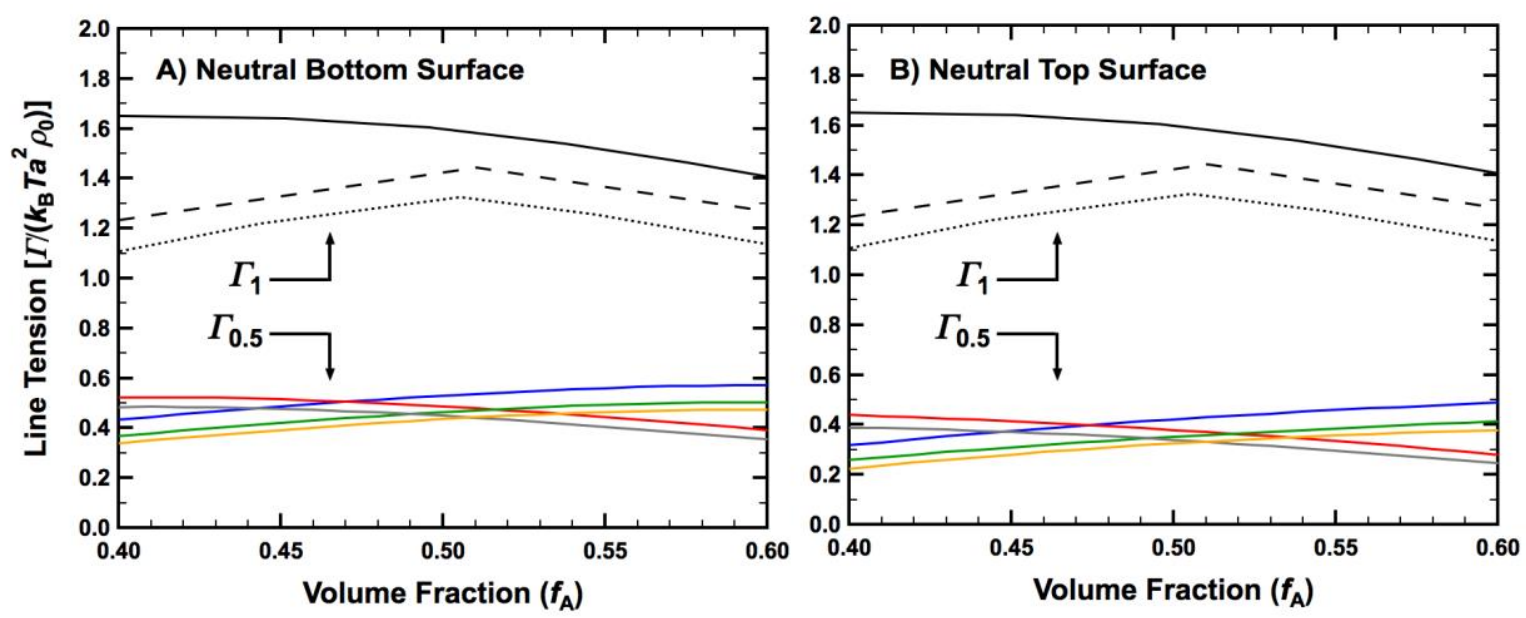

Figure 2: Line tensions of $0.5 L_{0}$ (colored curves) and $1 L_{0}$ (black curves) step edges calculated with SCFT for (a) neutral substrate with preferential free surface and (b) preferential substrate with neutral free surface. The blue, red, green, gray and yellow curves correspond to $m=0.5,1.0,1.5,2.0$ and 2.5, respectively, while the solid, dashed, and dotted black curves correspond to $m=0.5,1.0$ and 1.5 , respectively. The latter curves are adapted from reference 12 .

\section{Meta-stability}

PS-PTMSS $\left(t_{0}=1.65 L_{0}\right)$ was annealed at $180^{\circ} \mathrm{C}$ for various times on substrate surfaces comprising cross-linked blends of poly(4-tert-butylstyrene-random-methyl methacrylate-random-4-vinylbenzylazide) (PtBS-r-PMMA-r-PVBzAz) containing different constituent ratios. (The free surface is highly PTMSS preferential in these experiments.) Optical micrographs (Figure 3) reveal that the resulting block copolymer topography is highly dependent on surface composition. The two extremes, $48 \mathrm{~mol} \%$ and $65 \mathrm{~mol} \%$ PtBS are clearly preferential for PS and PTMSS blocks, respectively, as deduced from the 
formation of stable $1 L_{0}$ islands and holes. A composition intermediate to the two extremes ( $52 \mathrm{~mol} \%$ ) is seemingly neutral as evidenced by the formation of stable $0.5 L_{0}$ islands at all annealing times (10-180 min), in agreement with the theory presented in the previous section of this manuscript. Remarkably, compositions skewed slightly away from neutral towards preferential interactions $(49,50,55$, and $58 \mathrm{~mol} \%)$ exhibit markedly different behavior. Early times (circa $10 \mathrm{~min}$ ) generate mostly half islands as expected for a single neutral surface, but heterogeneous secondary nucleation and coalescence of different topography competes during extended annealing, causing significant overall surface restructuring. (Additional supplemental optical micrographs are shown in Figures S7-S8.) This thermodynamic metastability on near neutral surfaces and the factors governing reorganization were subsequently analyzed in detail; the origin of the labels superposed on Figure 3, defined relative to the initially-formed matrix thickness coincident with $0.5 L_{0}$ topography nucleation, will become clear in the next section. Additional analysis is postponed until the Discussion. 


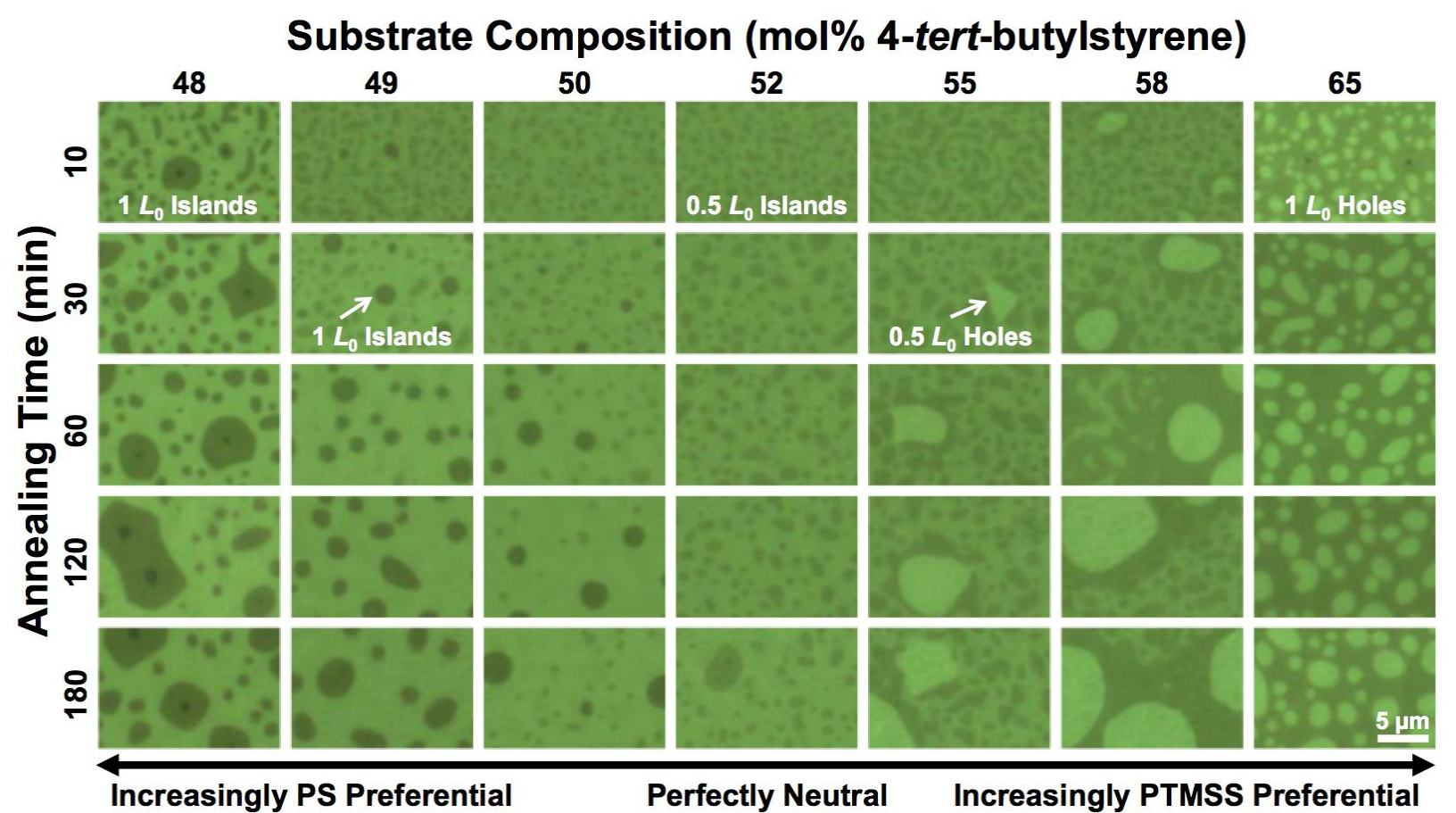

Figure 3: Optical micrographs demonstrate topographic formation $\left(t_{0}=1.65 L_{0}\right.$, annealed at $180^{\circ} \mathrm{C}$ ) and stability depend on substrate surface composition. See the Supporting Information for details on the blends used to tune composition. The scale bar is valid for all micrographs.

\section{Surface Restructuring}

The origins of metastability and the type of topography formed during secondary nucleation were studied with two boundary conditions: (1) slightly asymmetric wetting imposed by a moderately PS-preferential substrate paired with air or vacuum at the free surface (which are both highly PTMSS preferential), and (2) slightly symmetric wetting arising from a preferential substrate (cross-linked PS homopolymer) with a moderately PSpreferential top coat. 
Asymmetric wetting

PS-PTMSS with $t_{0}=1.35 L_{0}$ generates half holes in $10 \mathrm{~min}$, comprising a matrix at $t=$ $1.5 L_{0}$ and troughs at $t=1 L_{0}$ (Figure 4A). Continued thermal annealing (300 min total) further nucleates $1 L_{0}$ deep holes with a matrix still at $t=1.5 L_{0}$ and troughs now occupying $t=0.5 L_{0}$. Figure $4 \mathrm{~B}$ shows analogous experiments conducted with $t_{0}=1.65 L_{0}$; half islands form, again with a matrix residing at $t=1.5 L_{0}$ and apexes now at $t=2 L_{0}$. The sample annealed for 10 min already contains a low areal density of $1 L_{0}$ structures spanning $t=$ 1.5-2.5 $L_{0}$, which significantly grow in both areal density and footprint after extended annealing to $300 \mathrm{~min}$.

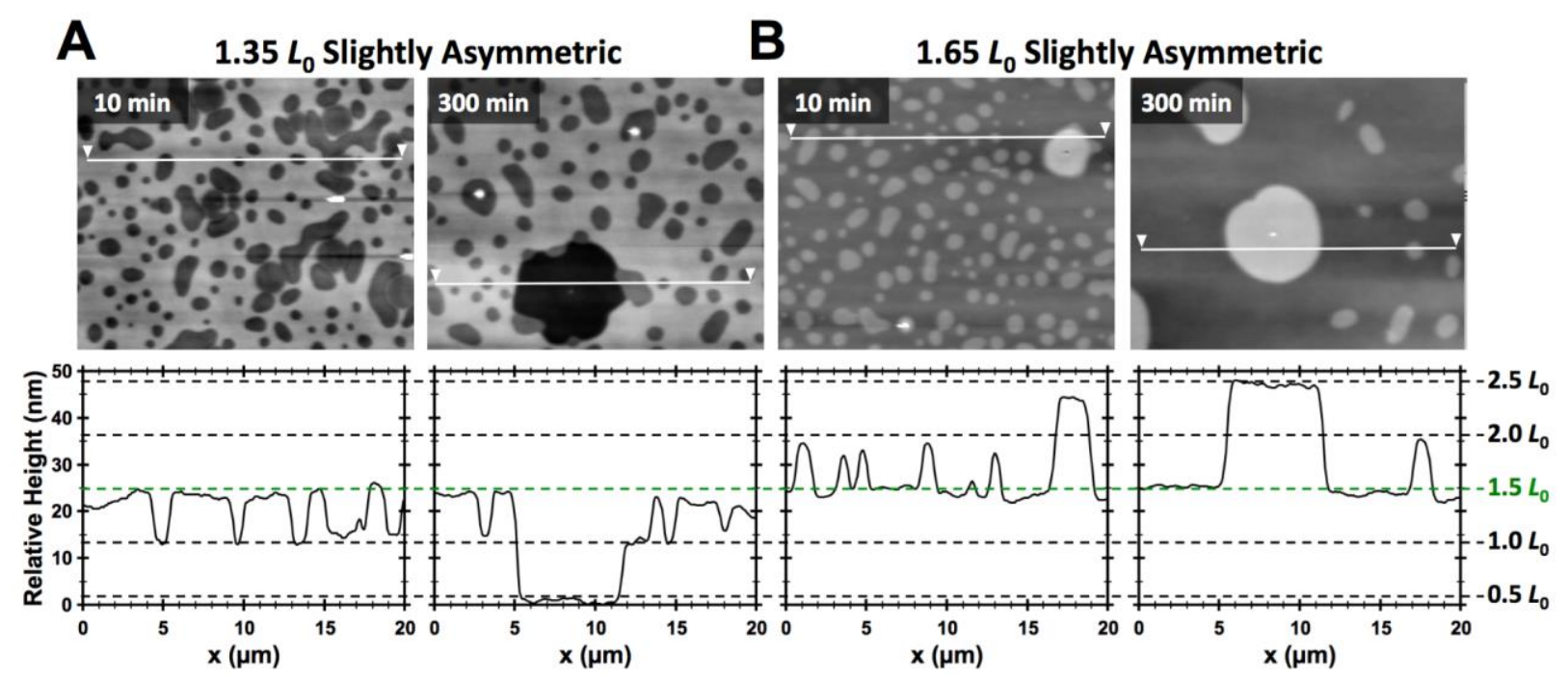

Figure 4: PTMSS-PS annealed at $180^{\circ} \mathrm{C}$ for various times on a near neutral substrate surface with air at the free surface.

Both $t_{0}=1.85 L_{0}$ and $2.15 L_{0}$ as-cast thicknesses also quickly form $0.5 L_{0}$ topography that slowly reconfigures into $1 L_{0}$ topography (Figure 5). Over time, the $t_{0}=1.85 L_{0}$ sample originally containing only half holes further nucleates half islands from the matrix region 
that span $t=2-2.5 L_{0}$ thicknesses. The $t_{0}=2.15 L_{0}$ sample, initially displaying half islands, also nucleates the opposite type of half topography ( $0.5 L_{0}$ holes) from the matrix region after 300 min annealing. For both samples, the mixture of nominally two different $0.5 L_{0}$ structures actually represents a transient state captured during surface reconstruction that will eventually yield $1 L_{0}$ topography spanning $t=1.5-2.5 L_{0}$ absolute film thicknesses. Further evidence supporting this conclusion is provided below. The $1 L_{0}$ topography formed after extended thermal annealing for all four samples shown in Figures 4 and 5 is consistent with asymmetric wetting exhibiting commensurability at $t=(n+0.5) L_{0} ; 1 L_{0}$ thick topography resides at $t=0.5,1.5$, and $2.5 L_{0}$ depending on the as-cast $t_{0}$. Additional film thicknesses $\left(t_{0}=1.17,2.35,2.65\right.$, and $2.85 L_{0}$, see Figures $\left.S 9-S 12\right)$ are fully consistent with the above interpretations.

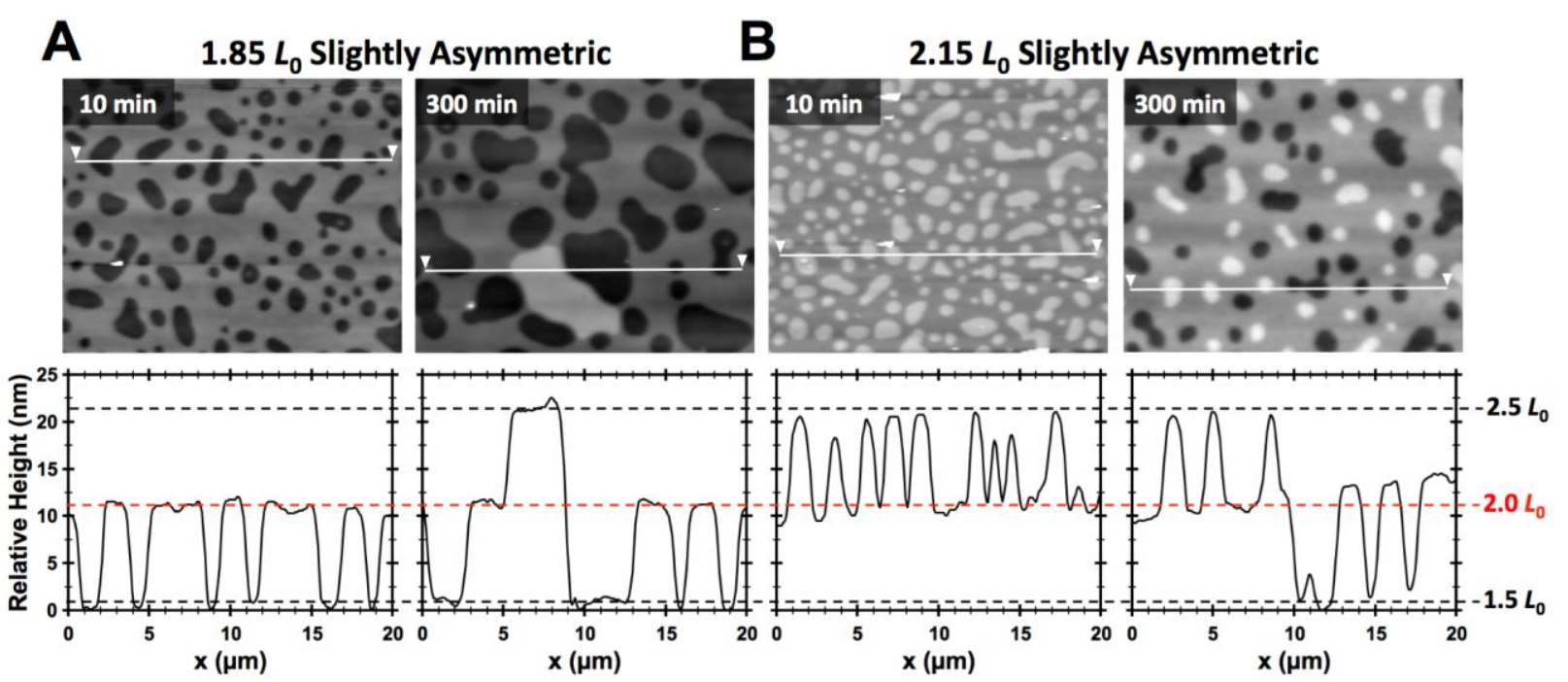

Figure 5: PTMSS-PS annealed at $180^{\circ} \mathrm{C}$ for various times on a near neutral substrate surface with air at the free surface. 
Symmetric wetting

Figures 6 and 7 show experiments conducted with a PS-preferential substrate surface and a near neutral top coat. At short annealing times, $t_{0}=1.31 L_{0}$ forms exclusively $0.5 L_{0}$ holes. (Note that the two-dimensional pattern looks nearly bicontinuous due to the large $\Delta t_{0}$ relative to the $t=1.5 L_{0}$ commensurate condition.) After extended annealing, 0.5 $L_{0}$ islands nucleate from the $t=1.5 L_{0}$ matrix. Evidence that these structures in fact represent $1 L_{0}$ topography spanning $t=1-2 L_{0}$ can be found by inspecting the $t_{0}=1.67 L_{0}$ sample (Figure 6B). At short annealing times (10 min), half islands form with a matrix at $t=$ $1.5 L_{0}$ and apexes at $t=2 L_{0}$, consistent with non-preferential wetting at the top interface. Further annealing (300 $\mathrm{min}$ ) of the same sample nucleates half hole-like features from the matrix, with troughs residing at $t=1 L_{0}$ thickness, and continued annealing to 600 minutes completely eliminates the matrix originally residing at $t=1.5 L_{0}$; the film now contains only $1 L_{0}$-thick topography spanning $t=1-2 L_{0}$ absolute film thicknesses. The final structures formed are thus consistent with full holes as expected for $1.5<t_{0} / L_{0}<2$ with symmetric boundary conditions characterized by commensurability at $t=n L_{0}$. Not all films fully transitioned from kinetically-formed half structures to thermodynamically-stable $1 L_{0}$ topography after $600 \mathrm{~min}$ annealing, but all films at least partially reconstructed after modest (300 min) thermal treatment. We conclude that most data reported herein at 300 min represent a fleeting snapshot captured during surface reconstruction; presumably given enough time, these samples would fully transition to $1 L_{0}$ topography as observed with $t_{0}=1.67 L_{0}$. 


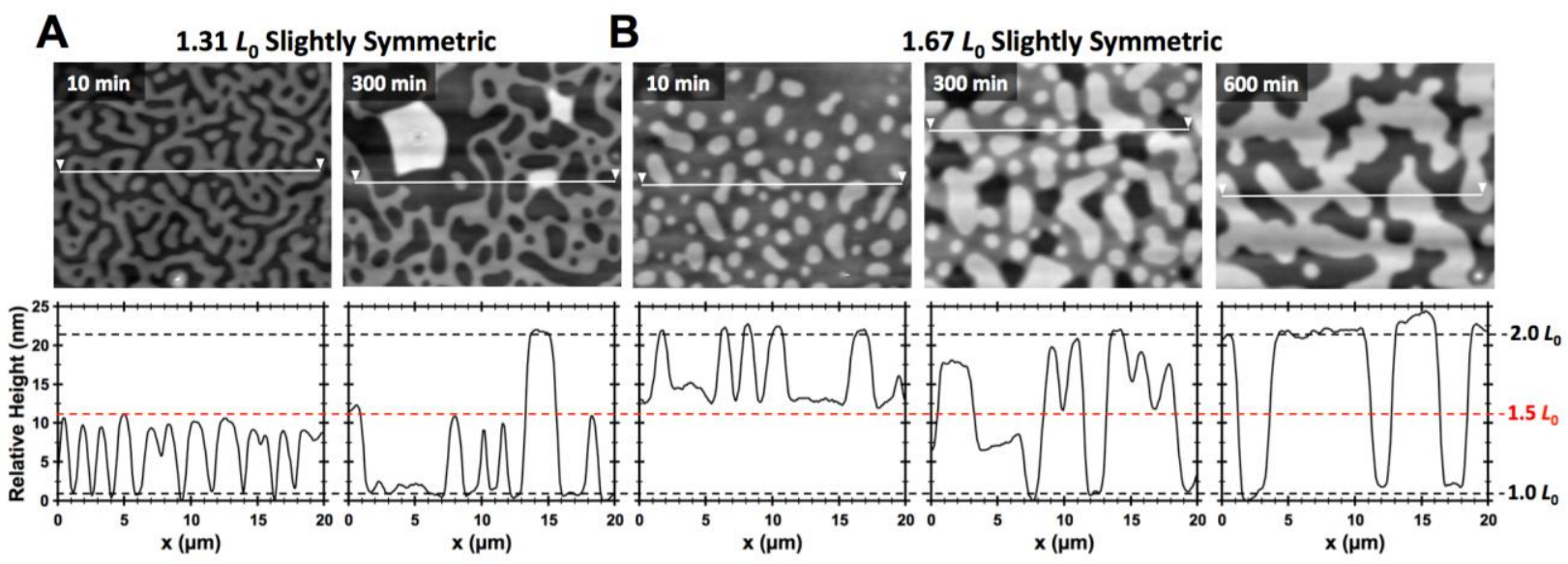

Figure 6: PTMSS-PS annealed at $180^{\circ} \mathrm{C}$ between a PS homopolymer substrate and a near neutral top coat.

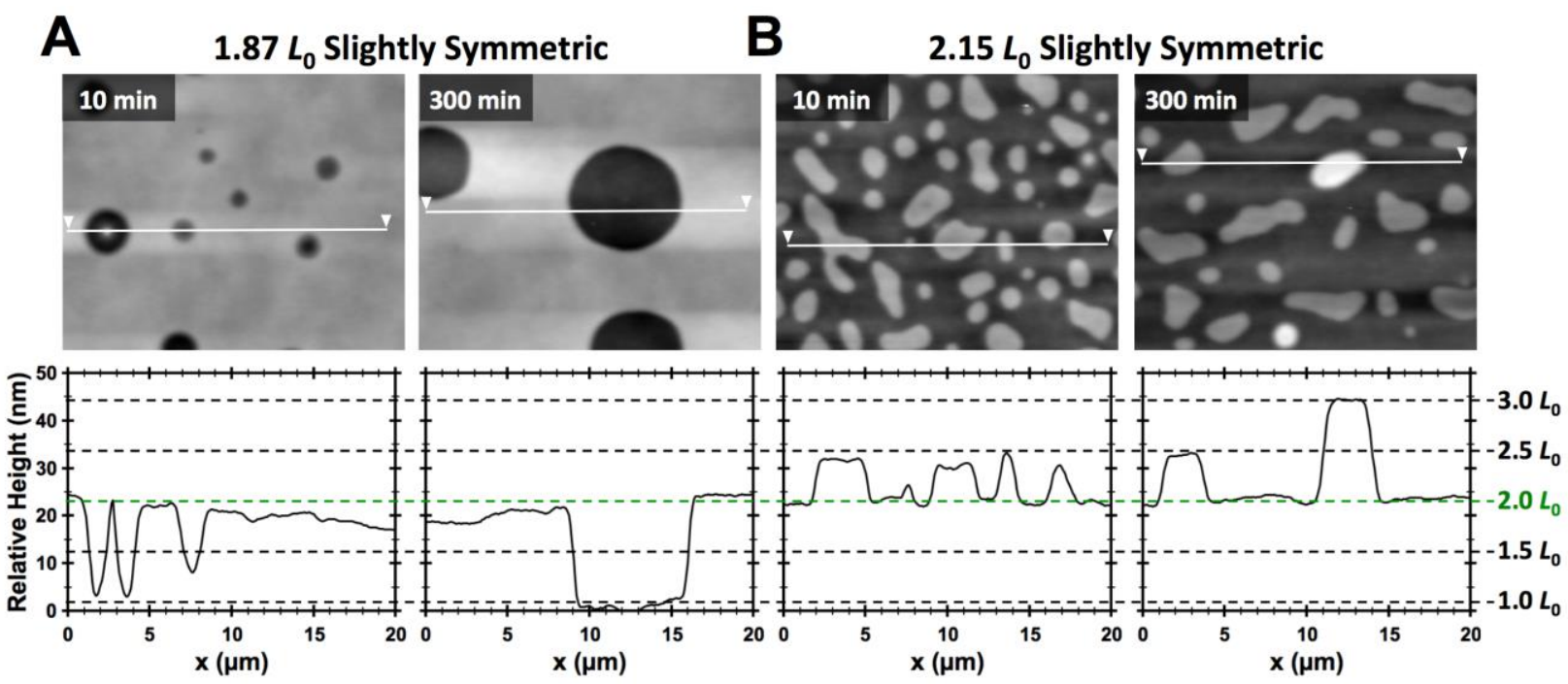

Figure 7: PTMSS-PS annealed at $180^{\circ} \mathrm{C}$ between a PS homopolymer substrate and a near neutral top coat.

A $t_{0}=1.87 L_{0}$ sample quickly forms a mixture of approximately $0.5 L_{0}$ and $1 L_{0}$ topography after $10 \mathrm{~min}$ annealing (Figure 7A). After $300 \mathrm{~min}$, the troughs of the $0.5 L_{0}$ features decrease in thickness, fully transforming into $1 L_{0}$ holes traversing $t=1-2 L_{0}$. 
Likewise, the half islands generated with $t_{0}=2.15 L_{0}$ (Figure 7B) begin to coexist with $1 L_{0}$ islands upon extended annealing. Additional samples with $t_{0}=1.16,2.37,2.63$, and $2.82 L_{0}$ are provided in the Supporting Information (Figures S13-S16) and generally reinforce the behavior observed in Figures 6 and 7.

\section{Discussion}

Previous studies on $0.5 L_{0}$ topography specifically targeted the use of a single "perfectly neutral" surface (characterized by exactly balanced interfacial interactions with each block, $\Delta \gamma \equiv \gamma_{\mathrm{AS}}-\gamma_{\mathrm{BS}}=0$ ) to generate half structures. ${ }^{7,8}$ While theory now confirms that half islands and holes are indeed thermodynamically stable in the presence of a single perfectly neutral interface, achieving true surface neutrality with real materials is technically impossible. Experimentally, interactions at both the bottom ${ }^{16-18}$ and top 8,19 surfaces are conveniently tuned with polymer composition. ${ }^{16}$ The experiments described in Figures 3-7 demonstrate that $0.5 L_{0}$ topography still kinetically forms on surface compositions that are near neutral, but thermodynamic metastability triggers surface reconstruction upon extended annealing. Quantifying surface neutrality (i.e., measuring $\mid \gamma_{\mathrm{s}}$ $-\gamma_{\text {neutral }}$, where $\gamma_{s}$ is the surface tension of the given surface and $\gamma_{\text {neutral }}$ is the surface tension of a perfectly neutral surface) with the precision necessary to differentiate small changes in surface composition is currently impossible, but Figure 3 provides qualitative insight into the breadth of the perfectly- and near-neutral composition windows. Care should be taken not to over-interpret the absolute magnitude of the differences; assuming surface energy scales linearly with polymer composition, the rate of change $(d \gamma / d c$, where $c$ is surface treatment composition) depends on the surface energy of the constituent monomeric 
components $\left(\gamma_{c=0}\right.$ and $\left.\gamma_{c=1}\right)$. BCP attributes such as relative volume fraction and the blockblock interfacial tension $\left(\gamma_{\mathrm{AB}} \sim \chi^{0.5}\right)$ may also contribute, although such conjectures remain speculative and currently unsubstantiated. The optical micrographs in Figure 3 do provide evidence that the timescale associated with nucleation $\left(\tau_{1}\right)$ and corresponding growth $\left(\tau_{2}\right)$ of secondary islands and holes after initial kinetic formation of $0.5 L_{0}$ topography $\left(\tau_{0}\right)$ correlates with the differential surface composition relative to perfect neutrality $\left(\tau_{1,2} \sim \Delta c^{\beta}\right.$ $=\left|c_{\mathrm{s}}-c_{\text {neutral }}\right|^{\beta}$. For instance, supposing a neutral surface treatment composition $c_{\text {neutral }} \approx 52$ mol $\%$ PtBS, $c_{s}=50$ and 55 mol\% show minimal evidence of secondary nucleation after 10 minutes, which begins to emerge only circa $30 \mathrm{~min}$. In contrast, surfaces with 49 and 58 mol\% already generate a low density of secondary nuclei after $10 \mathrm{~min}$, while surfaces far from neutral compositions entirely forego nucleation of metastable $0.5 L_{0}$ topography even at short times in favor of classical $1 L_{0}$ islands ( $48 \mathrm{~mol} \%$ ) and holes (65 mol\%). Extracting the exact functional form of $\tau_{1,2}$, including any exponential dependence $(\beta)$, would require additional kinetic studies beyond the scope of this report. Given the apparent $\tau$ dependence for obliteration of kinetically-formed $0.5 L_{0}$ topography, even definitive proclamation of perfect neutrality at $c_{\text {neutral }}=52 \mathrm{~mol} \%$ is strenuous at best, since lengthened annealing may ultimately yield surface reconfiguration.

At least some near neutral surface compositions displaying thermodynamic metastability are for all practical purposes "neutral" in the sense that they successfully orient block copolymer lamellae perpendicular to the substrate when coupled with a second neutral surface. ${ }^{20}$ Such orientation can only be achieved in the presence of two sufficiently neutral surfaces. ${ }^{21,22}$ Potentially subtle distinctions between perfectly neutral, near neutral, and preferential surfaces, straddling compositions capable and incapable of 
orienting BCPs, is critical for interfacial design. The surface restructuring mechanism studied in detail herein portends a trivial experimental approach that for the first time delineates perfectly neutral and near neutral surfaces. This demarcation might gain additional importance in the continued race towards high- $\chi$ BCPs for lithographic applications. $^{23}$

The surface reconstruction mechanism can be rationalized with commensurability effects established by surface boundary conditions operating on kinetically-formed $0.5 L_{0}$ topography. A single near neutral surface unambiguously generates $0.5 L_{0}$ topography at short annealing times $\left(\tau_{0}\right)$, as evidenced by Figures 3-7 and previous literature reports. 7,8 Subsequent restructuring depends on both the commensurability of the resulting matrix and the thickness occupied by half feature apexes/troughs (illustrated with select examples in Figure 8). For circumstances involving a matrix that is commensurate with the boundary conditions (e.g., Figures 4 and 7), $0.5 L_{0}$ topography transitions directly into $1 L_{0}$ topography either via mass transfer into (or out of) the existing $0.5 L_{0}$ structures or by direct nucleation of full features from the matrix (with a corresponding reduction of each half feature area footprint). Half structures thus appear to directly morph into full features during annealing, as depicted in Figure 8B. In contrast, when the matrix initially adopts a thickness that is incommensurate with the boundary conditions (e.g., Figures 5 and 6), it nucleates the opposite $0.5 L_{0}$ topography while simultaneously maintaining the original half features (Figure 8A). Pairs of 0.5 islands and holes eventually agglomerate, concurrent with annihilation of the matrix, to eventually yield $1 L_{0}$ topography terminating at thicknesses fully commensurate with the surface-imposed boundary conditions (Figure 6B). Note that Figure 8 depicts a preferential substrate and near neutral top coat in direct 
analogy to Figure 6B; see Figure S17 for the opposite illustration with a preferential top surface and near neutral substrate. We are unaware of any analogous studies describing the reorganization of $1 L_{0}$ topography driven by changes in surface wetting, although the results reported herein indicate such reconstruction may be possible with suitable materials that controllably alter preferential interfacial interactions. Top coats might facilitate the prerequisite dynamic wettability since they are inherently strippable using solvent without damaging the underlying BCP film. Alternatively, photo-induced switching of surface wettability may provide similar control via a complementary mechanism..$^{24,25}$

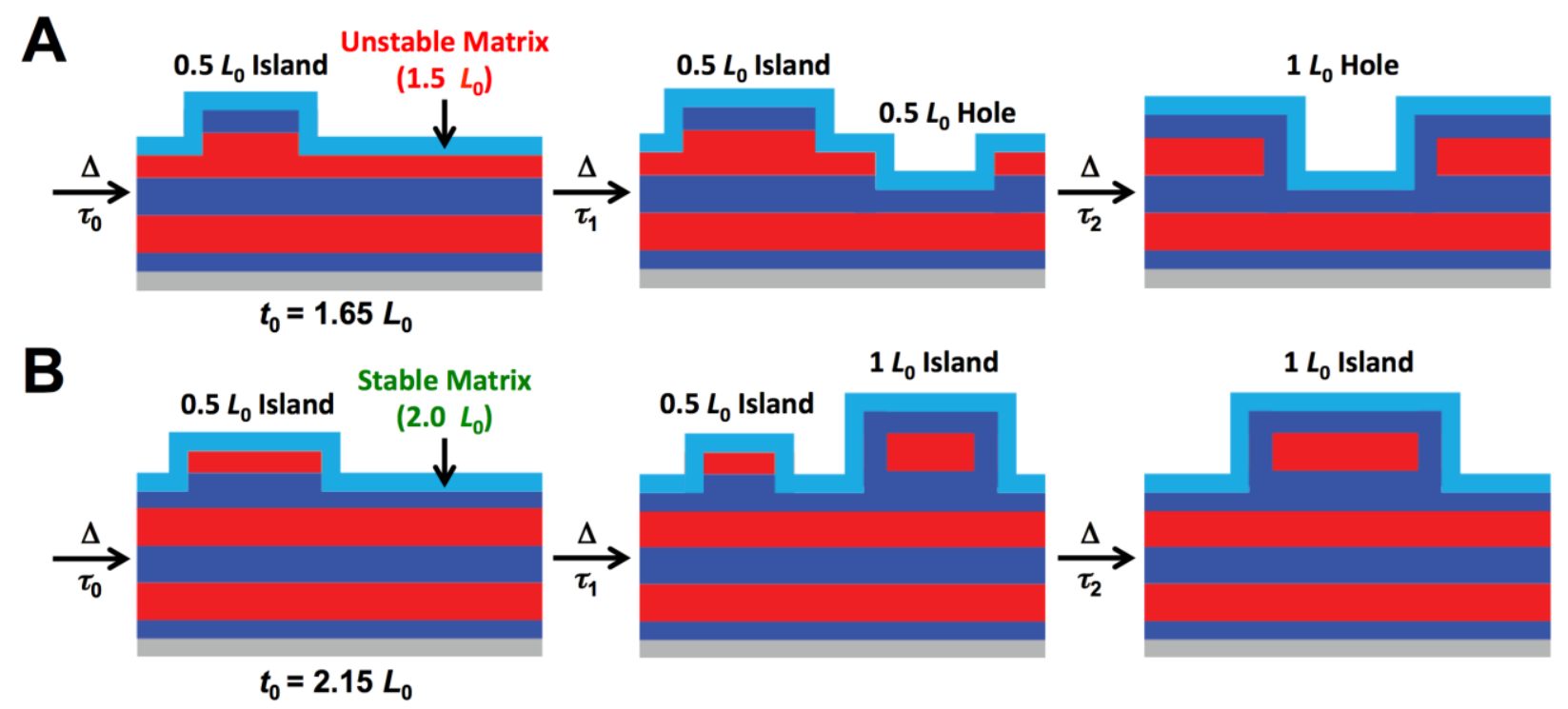

Figure 8: Surface reconstruction as a function of time for a preferential substrate and near neutral top coat (overall, slightly symmetric wetting) with as-cast BCP thickness A) $t_{0}=$ $1.65 L_{0}$ and B) $t_{0}=2.15 L_{0}$.

A brief discussion is warranted regarding the confinement regime accessed with maleic anhydride top coats. Koneripalli et al. have previously studied block copolymers 
sandwiched between a solid substrate and polystyrene top coat. ${ }^{26}$ Notably, with their materials, island and hole formation is suppressed in favor of stretched or compressed block copolymer domains that uniformly cover the as-cast thickness with an integral number of periods. The underlying physical characteristics that distinguish polystyrene from maleic anhydride top coats are still not fully understood. Both "hard" polystyrene top coat $\left(T_{\mathrm{g}} \approx 100^{\circ} \mathrm{C}\right)$ and apparently "soft" maleic anhydride top coats $\left(T_{\mathrm{g}} \approx 200^{\circ} \mathrm{C}\right)$ are nominally glassy under the selected annealing conditions (which differ between reports) with presumably typical elastic moduli circa $1 \mathrm{GPa}$. (Note that we have not measured the mechanical properties of maleic anhydride copolymers.) We speculate that the important difference may be related to the presence of trimethylammonium ions. For solubility reasons, our top coats are cast as trimethylammonium salts which upon annealing ringclose to form maleic anhydride with concomitant liberation of water and trimethylamine gas, ${ }^{8}$ likely forming internal voids within the top coat. Our working hypothesis is that these voids increase free volume, transforming the top coat from a glassy to melt state. Although we are not absolutely certain of this, there is no doubt these polymers can be considered "soft" for all practical purposes on the timescale of our experiments. Maleic anhydride top coats unambiguously contort to reveal block copolymer surface topography, which is readily interpreted to extract surface wetting information. ${ }^{8}$ In contrast, hard materials like polystyrene ${ }^{26}$ and $\mathrm{SiO}_{\mathrm{x}}{ }^{27}$ are incapable of deformation and consequently perturb the bulk block copolymer periodicity in lieu of island and hole formation. We conclude that the regime probed herein, using the nomenclature of Koneripalli, must be considered "pseudoconfinement," in analogy to annealing with a free surface (e.g., air or vacuum). Although surprising, to date we have identified no significant differences between the 
surface topography produced from a block copolymer annealed under a maleic anhydride top coat or a free surface. Since the present manuscript focuses on wetting effects and associated surface reconfiguration on near neutral surfaces, a detailed physical model encapsulating all of the aforementioned factors governing top coat pseudoconfinement is well beyond the intended scope of this report.

\section{Conclusion}

The through-film structure, thermodynamic (meta)stability, and reconfiguration mechanism of $0.5 L_{0}$ block copolymer topography formed in the presence of one neutral and one preferential interface were studied through a combination of experiment and selfconsistent field theory. Kinetic formation of $0.5 L_{0}$ features at short annealing times is driven by significantly reduced line tension relative to $1 L_{0}$ structures. The edge dislocation necessarily generated by $0.5 L_{0}$ topography is pinned to the neutral or near neutral interface (either top or bottom), in contrast to $1 L_{0}$ features in which defects are generally embedded within the interior of the film. While the utilization of a single perfectly neutral surface renders $0.5 L_{0}$ topography thermodynamically stable, a single near neutral surface imparts metastability relative to $1 L_{0}$ structures. Half features correspondingly reconstruct during annealing to nucleate and grow topography that ultimately transforms into $1 L_{0}$ islands or holes, determined by surface boundary conditions. Secondary nucleation timescales appear correlated with surface composition, whereby nearly neutral materials maintain metastable half features longer than surfaces skewed more preferential. The mechanism of surface reconstruction depends sensitively on film thickness and commensurability. 


\section{Materials and Methods}

\section{Experiment}

The BCP discussed herein, poly(styrene-block-4-trimethylsilylstyrene) (PS-PTMSS) with $L_{0}=22 \mathrm{~nm}$, typifies a convenient model system due to inherently PTMSS preferential interactions at the free surface ${ }^{28}$ which are readily modifiable with top coats. ${ }^{8}$ We note in passing that silicon-containing BCPs including PS-PTMSS are also potentially useful for lithographic applications. ${ }^{29-32}$ The influence of substrate composition on topographic metastability was studied with blends generated by mixing two poly(4-tert-butylstyrenerandom-methyl methacrylate-random-4-vinylbenzylazide) PtBS-r-PMMA-r-PVBzAz copolymers in various ratios, see the Supporting Information for details. Restructuring mechanistic studies applied various combinations of surfaces to systematically vary thin film commensurability conditions. The surfaces used included (1) a PtBS-r-PMMA-rPVBzAz near-neutral substrate surface treatment (slightly PS wetting) with 53 mol\% PtBS, 42 mol\% PMMA, 5 mol\% PVBzAz, (2) a near-neutral (slightly PS wetting) top coat, poly(styrene-alt-maleic anhydride)-random-poly(3,5-di-tert-butylstyrene-alt-maleic anhydride) (50 mol\% maleic anhydride, 20 mol\% 3,5-di-tert-butylstyrene, 30 mol\% styrene), and (3) a PS-preferential substrate surface, poly(styrene-random-4vinylbenzylazide) (95 mol\% PS, 5 mol\% PVBzAz). Synthetic details and characterization of these materials can be found in the Supporting Information and elsewhere. ${ }^{8}$

Theory

To model the terraces and the step edges between them, we use self-consistent field theory (SCFT), which is regarded as the state-of-the-art for block copolymer melts. ${ }^{33,34}$ 
SCFT has recently been used to provide what is undoubtedly the most accurate theoretical treatment of $1 L_{0}$ step edges in block copolymer films. ${ }^{12}$ Here, we use the same approach for the $0.5 L_{0}$ step edges.

The diblock copolymers are modeled by flexible Gaussian chains of $N_{\mathrm{A}} \mathrm{A}$-type segments joined to $N_{\mathrm{B}}$ B-type segments. Both types of segments are defined to occupy an equal volume of $\rho_{0}^{-1}$ and assumed to have the same statistical segment length, $a$. The composition of the diblock is given by $f_{\mathrm{A}} \equiv N_{\mathrm{A}} / N$, where $N=N_{\mathrm{A}}+N_{\mathrm{B}}$. The immiscibility of the $\mathrm{A}$ and $\mathrm{B}$ segments is modeled by a point-like repulsion controlled by the standard Flory-Huggins interaction parameter, $\chi_{\mathrm{AB}}$. For flexible polymers, the hard substrate can be treated by imposing a reflecting boundary at $z=0.34,35$ As justified in Ref. 12, the soft free surface can be conveniently modeled by filling the space above the diblock copolymer film with homopolymers of infinite molecular weight. The tensions (i.e., $\gamma_{\mathrm{Ah}}$ and $\gamma_{\mathrm{Bh}}$ ) and affinity (i.e., $\Delta \gamma=\gamma_{\mathrm{Ah}}-\gamma_{\mathrm{Bh}}$ ) of the free surface are controlled by introducing interactions between the homopolymer and the A and B diblock components with interaction parameters, $\chi_{\mathrm{Ah}}$ and $\chi_{\mathrm{Bh}}$, respectively. Note that the top coats in our experiments actually involve two surfaces, one with the BCP and another with the air. However, for thin top coats of uniform thickness, both surfaces will have the same area and thus can be treated as a single surface with a net tension equal to the sum of the BCP/top coat and top coat/air surface tensions.

In SCFT, the molecular interactions experienced by the A, B and homopolymer segments are approximated by mean fields, $w_{\mathrm{A}}(\boldsymbol{r}), w_{\mathrm{B}}(\boldsymbol{r})$ and $w_{\mathrm{h}}(\boldsymbol{r})$, respectively. The calculation starts with appropriate initial guesses for the fields, where their minima correspond to the expected locations of the corresponding components. Given the fields, diffusion equations are solved for propagators from which the concentrations of the three 
components, $\phi_{\mathrm{A}}(\boldsymbol{r}), \phi_{\mathrm{B}}(\boldsymbol{r})$, and $\phi_{\mathrm{h}}(\mathbf{r})$, are calculated. The fields are then adjusted iteratively until they satisfy the self-consistent conditions,

$$
\begin{aligned}
& w_{\mathrm{A}}(\boldsymbol{r})=\chi_{\mathrm{AB}} N \phi_{\mathrm{A}}(\boldsymbol{r})+\chi_{\mathrm{Ah}} N \phi_{\mathrm{h}}(\boldsymbol{r})+\xi(\boldsymbol{r}) \\
& w_{\mathrm{B}}(\boldsymbol{r})=\chi_{\mathrm{AB}} N \phi_{\mathrm{B}}(\boldsymbol{r})+\chi_{\mathrm{Bh}} N \phi_{\mathrm{h}}(\boldsymbol{r})+\xi(\boldsymbol{r}) \\
& w_{\mathrm{h}}(\boldsymbol{r})=\chi_{\mathrm{Ah}} N \phi_{\mathrm{A}}(\boldsymbol{r})+\chi_{\mathrm{Bh}} N \phi_{\mathrm{B}}(\boldsymbol{r})+\xi(\boldsymbol{r})
\end{aligned}
$$

where $\xi(\boldsymbol{r})$ is a pressure field that enforces the incompressibility condition, $\phi_{\mathrm{A}}(\boldsymbol{r})+\phi_{\mathrm{B}}(\boldsymbol{r})+$ $\phi_{n}(r)=1$. Once the solution is obtained, the domains of the film are defined according to the maximum concentration among $\phi_{\mathrm{A}}(\boldsymbol{r}), \phi_{\mathrm{B}}(\boldsymbol{r})$ and $\phi_{\mathrm{h}}(\mathbf{r})$.

To calculate the equilibrium terraces, we solve the field equations for uniform solutions with different numbers of layers, where the fields and concentrations depend only on the $z$ coordinate normal to the substrate. The free energy curves of the different film thicknesses exhibit a sequence of local minimums, and the first-order transitions between them are located by performing double-tangent constructions. ${ }^{15}$ The step edge separating two coexisting terrace heights is obtained by simply allowing the fields to also vary in the one of the lateral directions. In the case of $1 L_{0}$ step edges, there is a metastable solution for each possible location of the dislocation within the film; the preferred stable location is the one that minimizes the free energy. For $0.5 L_{0}$ step edges, however, the only physical solution is the one with a dislocation at the neutral surface. To obtain the line tension of the step edge, $\Gamma$, we calculate the free energy of the SCFT solution containing the step edge and subtract off the free energy of the coexisting uniform terraces with relative surface areas given by the lever rule of the double-tangent construction (see ref 12).

To model a neutral substrate and a preferential free surface, we set $\chi_{\mathrm{Ah}} N=30$ and $\chi_{\mathrm{Bh}} N=50$, which gives a surface affinity for the A component (representing PTMSS and 
denoted by red). For a preferential substrate and a neutral free surface, we set $\chi_{\mathrm{Ah}} N=\chi_{\mathrm{Bh}} N$ $=30$ and restrict our attention to morphologies where the B component (representing PS and denoted by blue) covers the entire substrate. In all cases, we fix $\chi_{\mathrm{AB}} N=20$, which corresponds to a block copolymer melt of intermediate segregation. The one shortcoming

of the SCFT is that we cannot solve the field equations for realistic values of $\chi_{\mathrm{Ah}}$ and $\chi_{\mathrm{Bh}}$, and consequentially the step edges will be somewhat sharper than in experiment.12 Although this will underestimate the line tensions, $\Gamma_{0.5}$ and $\Gamma_{1}$, of the $0.5 L_{0}$ and $1 L_{0}$ step edges, respectively, SCFT should still provide a reasonable prediction of their ratio, $\Gamma_{0.5} / \Gamma_{1}$.

\section{ASSOCIATED CONTENT}

Supporting Information. Detailed experimental procedures, mathematical model for island and hole nucleation, and Figures S1-S17. This material is available free of charge via the Internet at http://pubs.acs.org.

\section{Acknowledgements}

The authors thank Nissan Chemical Industries, Lam Research, the ASTC, and the National Science Foundation (Grants EECS-1120823 and EEC-1160494) for financial support. MJM thanks the IBM Ph.D. Fellowship Program and the National Science Foundation Graduate Research Fellowship (Grant No. DGE-1110007) for financial support. MJM thanks Elizabeth Lofano for assistance with cross-sectional SEM. GB thanks the Paul D. Meek Endowed Graduate Fellowship in Engineering for support. CJE thanks the Welch Foundation (grant \#F-1709) for partial financial support. CMB thanks the University of 
California, Santa Barbara for financial support. GW thanks the Rashid Engineering Regents

Chair and the Welch Foundation (Grant \#F-1830) for partial financial support. Any

opinions, findings, and conclusions or recommendations expressed in this material are

those of the authors and do not necessarily reflect the views of the National Science

Foundation or the sponsors.

\section{References}

(1) Coulon, G.; Russell, T. P.; Deline, V. R.; Green, P. F. Surface-Induced Orientation of Symmetric, Diblock Copolymers: A Secondary Ion Mass-Spectrometry Study. Macromolecules 1989, 22, 2581-2589.

(2) Russell, T. P.; Coulon, G.; Deline, V. R.; Miller, D. C. Characteristics of the SurfaceInduced Orientation for Symmetric Diblock PS/PMMA Copolymers. Macromolecules 1989, 22, 4600-4606.

(3) Mansky, P.; Russell, T. P.; Hawker, C. J.; Pitsikalis, M.; Mays, J. Ordered Diblock Copolymer Films on Random Copolymer Brushes. Macromolecules 1997, 30, 68106813.

(4) Peters, R. D.; Yang, X. M.; Kim, T. K.; Sohn, B. H.; Nealey, P. F. Using Self-Assembled Monolayers Exposed to X-Rays to Control the Wetting Behavior of Thin Films of Diblock Copolymers. Langmuir 2000, 16, 4625-4631.

(5) Coulon, G.; Ausserre, D.; Russell, T. P. Interference Microscopy on Thin Diblock Copolymer Films. J. Phys. France 1990, 51, 777-786.

(6) Ausserre, D.; Chatenay, D.; Coulon, G.; Collin, B. Growth of Two Dimensional Domains in Copolymer Thin Films. J. Phys. France 1990, 51, 2571-2580.

(7) Kim, S.; Bates, C. M.; Thio, A.; Cushen, J. D.; Ellison, C. J.; Willson, C. G.; Bates, F. S. Consequences of Surface Neutralization in Diblock Copolymer Thin Films. ACS Nano 2013, 7, 9905-9919.

(8) Maher, M. J.; Bates, C. M.; Blachut, G.; Sirard, S.; Self, J. L.; Carlson, M. C.; Dean, L. M.; Cushen, J. D.; Durand, W. J.; Hayes, C. O.; Ellison, C. J.; Willson, C. G. Interfacial Design for Block Copolymer Thin Films. Chem. Mater. 2014, 26, 1471-1479.

(9) Turner, M. S.; Maaloum, M.; Ausserre, D.; Joanny, J. F.; Kunz, M. Edge Dislocations in Copolymer Lamellar Films. J. Phys. II France 1994, 4, 689-702.

(10) Carvalho, B.; Thomas, E. Morphology of Steps in Terraced Block Copolymer Films. Phys. Rev. Lett. 1994, 73, 3321-3324.

(11) Liu, Y.; Rafailovich, M. H.; Sokolov, J.; Schwarz, S. A.; Bahal, S. Effects of Surface Tension on the Dislocation Structures of Diblock Copolymers. Macromolecules 1996, 29, 899-906.

(12) Stasiak, P.; McGraw, J. D.; Dalnoki-Veress, K.; Matsen, M. W. Step Edges in Thin Films of Lamellar-Forming Diblock Copolymer. Macromolecules 2012, 45, 9531-9538.

(13) Huang, E.; Pruzinsky, S.; Russell, T. P.; Mays, J.; Hawker, C. J. Neutrality Conditions for Block Copolymer Systems on Random Copolymer Brush Surfaces. 
Macromolecules 1999, 32, 5299-5303.

(14) Xu, T.; Hawker, C. J.; Russell, T. P. Interfacial Interaction Dependence of Microdomain Orientation in Diblock Copolymer Thin Films. Macromolecules 2005, 38, 2802-2805.

(15) Matsen, M. W. Thin Films of Block Copolymer. J. Chem. Phys. 1997, 106, 7781-7791.

(16) Mansky, P.; Liu, Y.; Huang, E.; Russell, T. P.; Hawker, C. Controlling Polymer-Surface Interactions with Random Copolymer Brushes. Science 1997, 275, 1458-1460. Ryu, D. Y.; Shin, K.; Drockenmuller, E.; Hawker, C. J.; Russell, T. P. A Generalized Approach to the Modification of Solid Surfaces. Science 2005, 308, 236-239.

Bang, J.; Bae, J.; Löwenhielm, P.; Spiessberger, C.; Given-Beck, S. A.; Russell, T. P.; Hawker, C. J. Facile Routes to Patterned Surface Neutralization Layers for Block Copolymer Lithography. Adv. Mater. 2007, 19, 4552-4557.

Bates, C. M.; Seshimo, T.; Maher, M. J.; Durand, W. J.; Cushen, J. D.; Dean, L. M.; Blachut, G.; Ellison, C. J.; Willson, C. G. Polarity-Switching Top Coats Enable Orientation of Sub-10-nm Block Copolymer Domains. Science 2012, 338, 775-779. Durand, W. J.; Carlson, M. C.; Maher, M. J.; Blachut, G.; Santos, L. J.; Tein, S.; Ganesan, V.; Ellison, C. J.; Willson, C. G. Experimental and Modeling Study of Domain Orientation in Confined Block Copolymer Thin Films. Macromolecules 2016, 49, 308-316.

(21) Turner, M. S. Equilibrium Properties of a Diblock Copolymer Lamellar Phase Confined Between Flat Plates. Phys. Rev. Lett. 1992, 69, 1788-1791.

(22) Walton, D. G.; Kellogg, G. J.; Mayes, A. M.; Lambooy, P.; Russell, T. P. A Free Energy Model for Confined Diblock Copolymers. Macromolecules 1994, 27, 6225-6228.

(23) Bates, C. M.; Maher, M. J.; Janes, D. W.; Ellison, C. J.; Willson, C. G. Block Copolymer Lithography. Macromolecules 2014, 47, 2-12.

(24) Maher, M. J.; Bates, C. M.; Blachut, G.; Carlson, M. C.; Self, J. L.; Janes, D. W.; Durand, W. J.; Lane, A. P.; Ellison, C. J.; Willson, C. G. Photopatternable Interfaces for Block Copolymer Lithography. ACS Macro Lett. 2014, 824-828.

(25) Lane, A. P.; Maher, M. J.; Willson, C. G.; Ellison, C. J. Photopatterning of Block Copolymer Thin Films. ACS Macro Lett. 2016, 460-465.

(26) Koneripalli, N.; Singh, N.; Levicky, R.; Bates, F. S.; Gallagher, P. D.; Satija, S. K. Confined Block Copolymer Thin Films. Macromolecules 1995, 28, 2897-2904.

(27) Lambooy, P.; Russell, T.; Kellogg, G.; Mayes, A.; Gallagher, P.; Satija, S. Observed Frustration in Confined Block Copolymers. Phys. Rev. Lett. 1994, 72, 2899-2902.

(28) Andersen, T. H.; Tougaard, S.; Larsen, N. B.; Almdal, K.; Johannsen, I. Surface Morphology of PS-PDMS Diblock Copolymer Films. J. Electron Spectrosc. Relat. Phenom. 2001, 121, 93-110.

(29) Maher, M. J.; Rettner, C. T.; Bates, C. M.; Blachut, G.; Carlson, M. C.; Durand, W. J.; Ellison, C. J.; Sanders, D. P.; Cheng, J. Y.; Willson, C. G. Directed Self-Assembly of Silicon-Containing Block Copolymer Thin Films. ACS Appl. Mater. Interfaces 2015, 7, 3323-3328.

(30) Jung, Y.-S.; Ross, C. A. Orientation-Controlled Self-Assembled Nanolithography Using a Polystyrene-Polydimethylsiloxane Block Copolymer. Nano Lett. 2007, 7, 2046-2050.

(31) Bita, I.; Yang, J. K. W.; Jung, Y.-S.; Ross, C. A.; Thomas, E. L.; Berggren, K. K. Graphoepitaxy of Self-Assembled Block Copolymers on Two-Dimensional Periodic 
Patterned Templates. Science 2008, 321, 939-943.

(32) Cushen, J. D.; Wan, L.; Blachut, G.; Maher, M. J.; Albrecht, T. R.; Ellison, C. J.; Willson, C. G.; Ruiz, R. Double-Patterned Sidewall Directed Self-Assembly and Pattern Transfer of Sub-10 nm PTMSS-b-PMOST. ACS Appl. Mater. Interfaces 2015, 7, 13476-13483.

(33) Fredrickson, G. H. The Equilibrium Theory of Inhomogeneous Polymers; Oxford University Press: Oxford, 2006.

(34) Matsen, M. W. Self-Consistent Field Theory and Its Applications. In Soft Matter, Volume 1: Polymer Melts and Mixtures; Gompper, G.; Schick, M., Eds.; Soft Matter; Wiley: Weinheim, Germany, 2006; pp. 87-178.

(35) Silberberg, A. Distribution of Conformations and Chain Ends Near the Surface of a Melt of Linear Flexible Macromolecules. J. Colloid Interface Sci. 1982, 90, 86-91. 
For Table of Contents use only

\section{Thin Film Surface Restructuring}

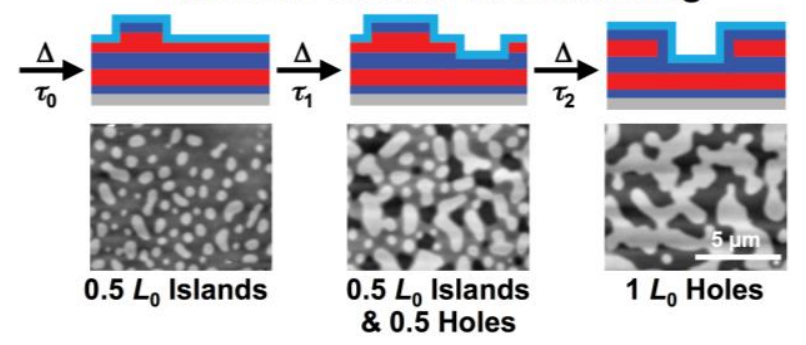

